\title{
Experimental evolution: experimental evolution and evolvability
}

\author{
N Colegrave ${ }^{1}$ and S Collins ${ }^{1,2}$ \\ ${ }^{1}$ Institute of Evolutionary Biology, School of Biological Sciences, University of Edinburgh, Edinburgh, UK and ${ }^{2}$ Max Planck Institute for \\ Plant Breeding Research, Cologne, Germany
}

\begin{abstract}
The suggestion that there are characteristics of living organisms that have evolved because they increase the rate of evolution is controversial and difficult to study. In this review, we examine the role that experimental evolution might play in resolving this issue. We focus on three areas in which experimental evolution has been used previously to examine questions of evolvability; the evolution of mutational supply, the evolution of genetic exchange and the evolution of genetic
\end{abstract}

architecture. In each case, we summarize what studies of experimental evolution have told us so far and speculate on where progress might be made in the future. We show that, while experimental evolution has helped us to begin to understand the evolutionary dynamics of traits that affect evolvability, many interesting questions remain to be answered. Heredity (2008) 100, 464-470; doi:10.1038/sj.hdy.6801095; published online 23 January 2008

Keywords: experimental evolution; evolvability; mutator; sex; robustness

\section{Introduction}

Natural selection produces organisms that are well adapted to their environment and the explanation of how complex adaptation may arise has been one of the great successes of the neo-Darwinian synthesis. There is little doubt that the functional significance of traits as diverse as the clutch size of great tits and the infanticide behaviour of male lions can be explained in terms of their effect on an individual's genetic contribution to the future. But are there characteristics of organisms that function not to increase their fitness, but instead to increase evolvability? That is, are there traits that are selected and maintained because they increase the ability of a population to respond to natural selection? Increasingly, the suggestion that many features of organismic design can only be understood in this context is taking hold (Kirschner and Gerhart, 1998; Earl and Deem, 2004). However, such claims are controversial and clear evidence has been hard to come by (Poole et al., 2003; Sniegowski and Murphy, 2006). Indeed, even the term evolvability has been defined and used in different ways, from a technical measure of the amount of additive genetic variation within a population to the ability of a population to generate novel variation or acquire novel functions (Kirschner and Gerhart, 1998; Wagner, 2005; Sniegowski and Murphy, 2006). Here, we define evolvability as the ability of a population to both generate and use genetic variation to respond to natural selection and an evolvability trait as a character that is selected because of its effects on evolvability.

Correspondence: $\operatorname{Dr} N$ Colegrave, Institute of Evolutionary Biology, School of Biological Sciences, University of Edinburgh, Ashworth Labs, King's buildings, Edinburgh EH9 3JT, UK.

E-mail:n.colegrave@ed.ac.uk

Received 16 May 2007; revised 12 October 2007; accepted 28 October 2007; published online 23 January 2008
Many features of organisms have been proposed as evolvability traits, and most can be loosely classified into one of the three categories. First, there are characters that directly increase the input of genetic variation. The elevated mutation rate observed in many species of bacteria is one obvious example. Similarly, there is some evidence that the existence of hypermutable domains within the genomes of pathogenic bacteria and viruses may allow more rapid adaptive responses in the face of host immune pressure (Moxon et al., 1994; Earl and Deem, 2004). Second, there are characters that increase genetic variation by mixing genetic material from different lineages. The widespread existence of eukaryotic sex has been explained in this context, but genetic exchange by various mechanisms is also common place among non-eukaryotes (Redfield, 2001). Third, there are characters that increase evolvability by altering the link between genotype and phenotype and so modulating the way in which a given amount of genetic variation is expressed at the phenotype level. This category includes a diverse array of organismic features that are less easily characterized than the previous two. The modularity of organismic design, the structure of gene networks and genetic architecture and the robustness of developmental mechanisms are examples of characters that may increase evolvability in this way (Wagner and Altenberg, 1996; Kirschner and Gerhart, 1998; Wagner, 2005; Hansen, 2006).

In theory, there is nothing to preclude the selection of traits that have no other effect than to increase the evolutionary potential of a population. However, in contrast to genes with direct effects on fitness, which respond directly to selection, evolvability genes are subject to indirect selection. Take as an example a gene that increases the genomic mutation rate, but has no other effect. Such a gene can potentially increase in frequency if it can 'hitchhike' with novel beneficial 
mutations that it creates (Sniegowski et al., 2000). Such indirect selection will be weak in comparison to direct selection, but even weak evolutionary forces can have considerable importance over the long timescales of evolutionary history.

However, while evolvability traits are possible in theory, demonstrating that a particular trait has been shaped by such selection pressures is far from straightforward. First, it is necessary to show that the trait really does increase the rate of adaptation. For example, increasing the supply of beneficial mutations could potentially increase the rate of adaptation, but may have little actual effect if adaptation is not limited by mutational supply. Second, it is also necessary to show that selection favours the trait because it increases evolvability rather than increased evolvability being an unselected by-product of selection for some other function (Sniegowski and Murphy, 2006). This distinction between the adaptive value of a trait and its unselected consequences is critical if we are to fully understand the forces that have moulded a trait (Williams, 1996).

Experimental evolution offers the potential to examine directly the evolutionary dynamics of putative evolvability traits. Such experiments allow researchers to observe directly whether a trait does indeed increase the rate of adaptation of a population, and if so, under what circumstances. In addition, it is possible to observe whether such traits increase in frequency in situations where the rate of adaptation is limiting (and perhaps equally importantly, that they do not increase in frequency when it is not). In this review, we will examine some of the ways in which the techniques of experimental evolution may improve our understanding of evolvability. We will not attempt a complete overview of the field. Instead, we begin by focusing on two areas, the evolution of elevated mutation rates in bacteria and the evolution of eukaryotic sex. These are areas in which our understanding of the evolutionary processes is reasonably well-developed, and experimental evolution studies have been central in aiding that development. We will then examine the much more controversial question of whether aspects of genetic architecture have evolved to increase evolvability (Wagner, 2005; Hansen, 2006). This is an area that has received a great deal of attention recently, but in which our understanding is much less well-developed. We will highlight how experimental evolution might help to develop this understanding.

\section{Mutational supply}

\section{Constitutive mutators}

Adaptive evolution ultimately depends on mutation to generate the genetic variation, that is, its fuel. Thus, it seems intuitive that an organism that increases its rate of mutation might benefit from increased evolvability. This intuition is supported by theory, at least under certain conditions. Population genetic models show that in a poorly adapted population, genes that increase the genomic mutation rate (termed mutators) can spread by hitchhiking with the beneficial mutations that they produce (Johnson, 1999a,b; Sniegowski et al., 2000; Tenaillon et al., 2000). However, this is much more likely to occur in organisms lacking recombination so that the association between mutator and mutation is not broken down, and is unlikely to be a significant force in sexual organisms.

Naturally occurring mutator strains are found in many bacteria, including Escherichia coli. Loss-of-function mutations in DNA repair systems means that these strains have a mutation rate 10- to100-fold higher than wild-type strains. This has allowed the evolutionary dynamics of mutator genotypes to be examined in the lab. Early experiments showed that chemostats inoculated with mixtures of mutator and wild-type $E$. coli would frequently become dominated by the mutator genotype (Cox and Gibson, 1974; Chao and Cox, 1983). The appearance and subsequent spread to fixation of mutator alleles within 3 of the 12 long-term selection lines of the Lenski's group (Sniegowski et al., 1997) showed that such genes can also spread when they arise naturally. Together these results provide direct evidence that genes for elevated mutation rate can spread through populations, and that they do so at a rate too high to be explained by genetic drift. However, is increased evolvability the selective force driving the increase in frequency of these mutators or is their spread due to some other direct selective benefit?

Further experiments cast doubt on a necessary link between elevated mutation rate and increased evolvability. When the rate of adaptation of mutator and wildtype E. coli was compared directly, elevated mutation rate had minimal effect on the rate of adaptation except in poorly adapted populations with small effective population sizes (de Visser et al., 1999). The rate of adaptation of larger populations was limited, not by the rate of mutational supply, but by the efficiency of selection, and so elevated mutation rates had little effect (Gerrish and Lenski, 1998; de Visser et al., 1999). Thus, increased mutational supply does not guarantee an increase in evolvability.

However, clear evidence that mutator genes can spread because they increase evolvability came from detailed examination of the three mutator lines from Lenski's experiments. In two of the three lines, the spread of the mutator genotype was accompanied by an increase in the rate of adaptation relative to populations that did not substitute mutator alleles (Shaver et al., 2002). In addition, there was no evidence that the increase in mutation rate seen was favoured by direct selection (Shaver et al., 2002). However, the observed benefits of increased mutation supply are short-lived. Comparison of the fitness of the mutator populations with non-mutator lines several thousand generations later showed no measurable affect of mutator fixation on the end fitness of a population (Shaver et al., 2002). This may not be that surprising, since all of the populations had become well adapted to the simple and unchanging laboratory environment by that point, and increased evolvability offers little benefit under such conditions (de Visser et al., 1999). However, this does mean that while increased evolvability may provide the adaptive explanation of why mutators increased in frequency, it does not explain their current maintenance within the populations. The question of why these populations remain fixed for mutators in the absence of any effect on current evolvability remains to be answered (Shaver et al., 2002).

Taken together, these studies provide support for the theoretical prediction that genes for elevated mutation 
rate can spread because they increase evolvability. However, they also show clearly how restrictive the conditions are in which this will occur and how transient such benefits can be. Such selection is unlikely to be particularly strong in natural populations, unless environmental change occurs at a high rate. This is in line with the fact that, while mutators in many natural populations of bacteria tend to be at low frequency, they are often found at much higher frequency in pathogenic bacteria (LeClerc et al., 1996; Oliver et al., 2000), which are likely to experience frequent bottlenecks and strong directional selection.

\section{Environment-specific mutators}

If advantages of increased mutation rate are likely to be transient in natural populations, then an organism that could increase its mutation rate with increased evolvability would be beneficial, but reduce it at other times might be at a selective advantage. Such environmentspecific modulation of mutation rates does occur in bacteria (Rosenberg et al., 1998; Metzgar and Wills, 2000; Bjedov et al., 2003). For example, when E. coli are starved, their rate of mutation increases, and the increase in beneficial mutation can allow them to survive the period of starvation (Rosenberg et al., 1998; Metzgar and Wills, 2000). The cellular machinery underlying these variable mutation rates has turned out to be remarkably complex, with several enzymes involved, allowing fine adjustments in mutation rate (Metzgar and Wills, 2000; Jarosz et al., 2007). The complexity of the machinery, combined with comparative evidence that it has been maintained over long evolutionary timescales and even duplicated (Erill et al., 2006) provides further evidence that the mutation rate variation is an adaptation, rather than a pathological consequence of the replication machinery breaking down under stress. However, whether it is an adaptation to increase evolvability is far from clear cut and alternative explanations have been proposed (Metzgar and Wills, 2000). In particular, the increased mutation rate might simply be a consequence of changes that allow the DNA replication system to copy badly damaged DNA or DNA that cannot otherwise be copied. Alternatively, if there is a trade-off between precision of replication and growth or survival in a stressful environment, the reduction in precision might represent a reallocation of resources. In either case, the increased evolvability would be regarded as a consequence rather than the selected function of the adaptation. Experimental evolution could potentially be used to disentangle these hypotheses. For example in E. coli, the elevated mutation rate is due to a switch from the standard polymerase enzyme to an error-prone DNA polymerase, which is also able to copy damaged DNA (Rosenberg et al., 1998; Metzgar and Wills, 2000). If it is possible to knock out this switch and manipulate the mutation rate of the standard polymerase enzymes, it will be possible to examine whether the elevated mutation rate is adaptive in the absence of an increase in the ability to copy damaged DNA. Given the success of experimental evolution in unravelling the selective forces acting on constitutive mutators, it seems likely that it will provide a powerful tool in understanding their facultative counterparts. Coming up with experimental protocols and techniques to disentangle the selected- versus-emergent functions of the machinery that allows organisms to fine-tune their mutation rates remains a challenge for the future.

\section{Genetic exchange}

\section{Eukaryotic sex}

Theoretical work (Gerrish and Lenski, 1998), as well as the experiments with bacteria discussed above (de Visser et al., 1999), demonstrates clearly that the rate of mutational supply will often not limit rates of adaptation. In particular, in large populations where abundant beneficial mutations are available, adaptation may be limited by the rate at which these beneficial mutations can be fixed. In asexual populations, beneficial mutations that arise in different lineages compete with one another, and cannot be fixed together (Gerrish and Lenski, 1998). This process of clonal interference is a potentially important limit on the rate of adaptation in asexual populations. The eukaryotic sexual cycle of meiosis and syngamy allows beneficial mutations that arise in different lineages to be brought together into the same individual, potentially removing the problem of clonal interference and increasing the efficiency of selection. Indeed, the machinery involved in meiosis and syngamy appears so obviously designed to increase the variation of offspring, that for many years, it was accepted without question that the function of sex was to increase the ability of a species to evolve. More recently, this explanation has been questioned since sex carries costs to the individual that would usually be expected to outweigh any benefits to the species (Maynard Smith, 1978; Bell, 1982). Nevertheless, more recent population genetic theory has shown that genetic modifiers for increased sex or recombination can increase in frequency because they increase the rate of adaptation (Charlesworth and Barton, 1996). The modifiers are able to spread in a similar fashion to the mutators discussed above, by hitchhiking with the higher fitness genotypes that they have created.

Experiments in facultative sexual eukaryotes show clearly that sex can increase the rate of adaptation in a novel environment. In Chlamydomonas reinhardtii, even a single round of sexual reproduction can increase the rate at which a population adapts to a novel environment in the short term (Colegrave et al., 2002), and longer term increases are observed after multiple rounds of sex (Colegrave, 2002; Kaltz and Bell, 2002). Similar results have been observed in yeast, where experimentally knocking out the sexual cycle causes populations to adapt less rapidly to harsh laboratory environments (Goddard et al., 2005). Evidence that these benefits come from bringing together beneficial mutations is because of the fact that the benefits of sex observed in yeast were not observed in populations of yeast (Goddard et al., 2005) or of $C$. reinhardtii (Renaut et al., 2006) grown in benign laboratory environments. Furthermore, clonal interference is expected to be strongest in large populations, and this is also where the benefits of sex in C. reinhardtii are greatest (Colegrave, 2002).

These experiments provide strong support that a function of eukaryotic sex is to increase the rate of adaptation to novel or changing environments. Whether this selective benefit of sex is enough in itself to outweigh 
its costs remains to be seen, and experiments that test this directly would be welcomed. Certainly, at least one experiment with yeast found benefits of sex in benign but not novel environments, supporting a role for sex in purging deleterious mutations (Zeyl and Bell, 1997). Evaluating the benefits of sex under different selective scenarios would appear to be a productive avenue for future research. Nevertheless, there is good reason to take seriously the idea that one of the most ubiquitous features of life may have evolved because of its effects on evolvability - a hypothesis that appears to be supported by comparative and macroevolutionary observations; asexuality is distributed across the tree of life, but it is rare to find higher taxa in which all species are asexual. In essence, asexual species tend to form twigs on the tree of life, rather than whole branches. Such a distribution is consistent with the idea that asexual lineages have short evolutionary lifetimes due to a lack of evolvability (Maynard Smith, 1978; Bell, 1982).

\section{Genetic exchange in non-eukaryotes}

Genetic exchange is not limited to eukaryotes and appears to be an ubiquitous feature of life. Evidence of gene transfer is present in virtually all bacterial genomes that have been examined (Narra and Ochman, 2006) and also occurs in both DNA and RNA viruses. However, the mechanisms of genetic exchange in non-eukaryotes are often involved in other processes, which provide alternative functional explanations. Genetic exchange in bacteria is frequently achieved via a third party such as a conjugative plasmid or a virus. In these cases, the exchange is probably a by-product or the reproductive strategy of this party. Bacterial transformation, where DNA is taken up from the environment and incorporated into the genome, would seem a more hopeful candidate, as no third party is involved. However, examination of machinery involved suggests that the main function of transformation is nutritional, with genetic exchange again relegated to a by-product (Redfield, 2001). The segmented genome of some RNA viruses allows genetic exchange to occur and has been shown experimentally to increase the rate of viral adaptation under certain circumstances (Poon and Chao, 2004). However, whether this is the function of segmentation, or whether some other function such as a reduction in packaging constraints or avoidance of the monocistronic messenger problem (Nee and Maynard Smith, 1990) remains an open question. Thus, it appears to us that genetic exchange outside eukaryotes is usually a by-product of other functions, and any increased evolvability that results is an unselected consequence.

\section{Evolvability and fitness landscapes}

The very idea that evolvability can be the target of natural selection supposes that some state or mechanism places a speed limit on adaptation. Fitness landscapes are produced by plotting fitness against genotype in a given environment and provide a useful framework within which the process of evolutionary change can be examined. At its simplest, an adapting population can be thought of as moving across this landscape stepping from genotypes of low fitness to genotypes of higher fitness and ultimately arriving at a local fitness optima, or adaptive peaks. The traits that we have discussed so far increase evolvability by allowing populations to climb adaptive peaks on stationary landscapes more rapidly, for example, by reducing the time until a genotype of higher fitness appear in the population. However, the topography of the landscape itself may constrain adaptation. In particular, if the landscape is rugged, such that high-fitness genotypes are frequently separated from other high-fitness genotypes by adaptive valleys containing low-fitness genotypes, the ability of natural selection to maximize fitness and reach the fittest genotype may be limited. In this case, an alternative way in which evolvability could be increased would be through changes in the shape or stability of the fitness landscape, which would allow population to move around the landscape more easily. In this case, evolvability would result from making fitness landscapes less rugged. This would entail changing how particular genotypes map to particular values of fitness by changing the interactions between components of fitness. Thus, changes in evolvability by selection on the shape of fitness landscapes could occur by making or destroying gene-gene interactions, and be detectable as changes in epistasis.

A mechanism that changes the ruggedness of fitness landscapes is implicit in much of the discussion of how the modularity at many levels of organismic organization may provide evolvability benefits, since increased modularity is expected to reduce the ruggedness of fitness landscapes (Wagner and Altenberg, 1996; Kirschner and Gerhart, 1998; Wagner, 2005; Hansen, 2006). However, can natural selection act directly on the shape of fitness landscapes to increase evolvability, or is the shape of the fitness landscape simply an unselected consequence of selection on other traits? As of yet, there are few clear answers to these questions. However, we believe that there are several ways in which experimental evolution can help us to address this fundamental question. While experimental evolution in bacteria has been used to examine the shapes of fitness landscapes (Travisano et al., 1995; Colegrave and Buckling, 2005), there has been little experimental investigation of if or how selection acts on their shape.

Experimental evolution can provide insight into the potential for this kind of selection by allowing us to examine constraints on landscapes directly. For example, the topography of fitness landscapes depends critically on the nature and size of epistatic interactions between loci, with greater epistasis leading to more rugged landscapes. Experiments with the goal of understanding how epistasis affects adaptation has focused on questions that can be applied to evolvability: does the degree of epistasis affect evolvability, is there heritable variance in epistasis that natural selection could act on and does natural selection actually act directly on evolvability by changing the degree of epistasis?

There is clear experimental evidence that epistasis affects evolvability. Recent work by Weinreich et al. (2006) showed that sign epistasis limits the number of accessible adaptive walks for a single protein, even when mutational supply is high enough that all single mutants occur. In addition, epistatic interaction have been shown to affect the rate of fitness recovery in viruses (Sanjuan et al., 2005) and E. coli (Moore et al., 2000), where negative epistasis speeds up the rate of fitness increase. This general effect is sensitive to mutational supply, where 
more epistasis speeds up fitness recovery in small populations, and intermediate levels of negative epistasis are favoured in larger populations. Sign epistasis also affects the order of fixation of beneficial mutations, and further constrains adaptation (Weinreich et al., 2006). Thus, epistasis can act as a strong constraint on evolvability, and that mutations that reduce the level of genetic interaction, such as the duplication of regulator genes, could in principle be favoured for this reason.

Changes in epistasis may make fitness landscapes less rugged or more changeable over time but since epistatic interactions have direct fitness effects, it is unclear whether selection is also acting directly on evolvability. Like other components of fitness that selection may act on, levels of epistasis have a distribution and are heritable (Martin et al., 2007). Experimental evolution could be used as a test of whether changes in epistasis correlate with changes in evolvability by seeing if mutations are fixed in more and less evolvable population samples from different parts of this distribution. Here, one would expect lower levels of epistasis in more evolvable populations that were adapted to randomly fluctuating environments if epistasis actually poses an important constraint on the number of possible adaptive solutions. The direct effect of epistasis on fitness increase could be measured, and evolvability of the end populations could be compared with the stability of their fitness landscapes by mutagenizing the populations or selecting them in a second novel environment. Experiments studying the correlation between robustness and the degree of antagonistic epistasis observed a correlation between the strength of epistatic interactions and the average effect of deleterious mutations (Wilke and Adami, 2001). Large-scale molecular genetics techniques are becoming more and more accessible, and can be used for experimental evolution. For example, it is possible to systematically create populations of microorganisms, such as yeast or bacteria, where individual genes could be duplicated. This would allow us to examine whether decreasing epistatic constraints by gene duplication increases evolvability. Overexpressing a single gene and comparing the rate of adaptation to cases where two copies of the gene are present could measure the effect of simply increasing the amount of gene product. Comparative studies of epistatic interactions or of gene networks between closely related species could be used to gain an idea of how common changes in epistatic relationships are over evolutionary timescales (Sanjuan and Elena, 2006).

In addition to characters such as epistatic interaction, that are intrinsic to the organisms under selection, specific types of environmental change or ecological interactions may favour changes in the ability to move on fitness landscapes. Here, experimental evolution can allow us to examine whether selection leads to changes in fitness landscape topography. The majority of work on this to date is in digital organisms, where elevated mutation rates result in types that occupy a lower but broader fitness peak and have the ability to displace populations on higher, narrower peaks (Wilke et al., 2001). Using digital organisms, the evolved populations have a high competitive fitness but low absolute fitness. If selection acts on changes in fitness landscape topography, nontransitive increases in fitness may be important, and measures of fitness using competition against ancestor would not be a very informative measure. Subsequent work investigating robustness has demonstrated that selection can alter the geometry of a fitness peak (Montville et al., 2005; Sanjuan et al., 2007), but no effort has been made to relate these studies to evolvability. These studies show that viruses with different mutation rates may provide a biological model for investigating changes in fitness landscape topography. A systematic comparison of different measures of fitness and estimates of nontransitive fitness increases in different experimental systems are needed if we are to understand the role of changes in fitness landscape topography in evolvability. In addition, it is unclear from experiments in digital organisms where mutation rates are held constant if selection in biotic systems would simply act to modify the mutation rate rather than the fitness landscape. In short, experimental versions of these simulations are needed.

Published studies hint that evolvability may be a target of natural selection, but none can convincingly rule out that it is simply a product of selection on other characters. Experimental evolution can help us begin to unravel whether traits such as mutational robustness are simply consequences of adaptation for ecological robustness, or whether they are selected directly. Since evolvability, like fitness, is an abstract property rather than a specific mechanism, determining if and how selection acts on evolvability will require synthesizing a number of studies that encompass different mechanisms, such as changes in mutation rates, recombination rates and degrees of epistasis. Such a synthesis requires some sort of unifying framework for deciding what constitutes an increase in evolvability, and looking at changes in fitness landscape topography and stability may provide such a unifying framework. Selection experiments can provide the means to study how selection may act on fitness landscapes, correlate this to subsequent changes in characters such as evolvability, and provide a mechanistic basis for the change in character (for example, a decrease in levels of epistasis).

\section{Concluding thoughts}

Experimental evolution is a powerful tool for examining whether processes predicted by theory can occur in real systems. Such experiments allow us to separate the possible from the merely plausible. We have seen that some traits, such as mutator strains in bacteria, can be selected because they increase evolvability, but that such selection is likely to be rare. In contrast, eukaryotic sexual reproduction appears to provide general adaptive benefits, and increased evolvability is at least a potential explanation for the ubiquity of sex in nature. Whether there are other features of the genetic architecture of organisms that can be explained in this way remains to be seen.

We have focused on understanding whether evolvability is itself being selected or whether it is an unselected consequence of selection for some other function. This is not because we view traits in the latter category as uninteresting or unimportant. It is simply that understanding why a trait exists requires investigating the selective forces that brought it about. Since evolvability traits are affected by indirect selective forces, which are less well understood than direct selection, we think that 
such traits are particularly interesting from an evolutionary point of view. Of course, even traits that lead to increased evolvability as a by-product of other functions may have profound consequences for evolutionary dynamics. Genetic exchange between bacteria has played a critical role in the evolution of resistance to antibiotics (Earl and Deem, 2004) and is likely to continue to do so too in the future. Similarly, understanding the implications of the highly mutable contingency loci in pathogens for drug design programs and future disease evolution is necessary, whatever their ultimate evolutionary function. We anticipate that experimental evolution combined with the increasing ability to genetically manipulate study organisms, creating variation in the traits of interest will continue to be useful in this context.

A major challenge for future research will be incorporating more realistic environments. In particular, previous experiments have typically dealt with simple forms of environmental change, where the environment changes dramatically and instantaneously and strong directional selection is applied for many generations. On a stationary rugged fitness landscape, this virtually guarantees that large populations will adapt rapidly and that replicate lineages will diverge. However, the kinds of environmental change experienced by real evolving populations will be more complex, and the process of adaptation, along with the benefits of increased evolvability are likely to depend very much on the details of environmental change. For example, recent simulation work has shown that including non-instantaneous rates of environmental change results in mutations of smaller effect being fixed and less divergence between adapted populations (Kopp and Hermisson, 2006; Collins et al., 2007). Similarly, the low frequency of constitutive mutators in natural bacterial populations compared with their fixation in laboratory populations may be due to frequent changes in the direction of selection in nature. While several researchers have begun to conduct experimental evolutionary studies in more natural settings (for example, Grimberg and Zeyl, 2005), experiments, which link such factors as the rate or degree of environmental change to the benefits of sex or mutator genes, will be invaluable in providing a more complete understanding of evolvability.

However, the area in which the most exciting progress seems likely to be made is in studies that combine experimental evolution with modern genomics. Technological advances, combined with the relative simplicity of organisms typically used in experimental evolution, mean that it is becoming increasingly feasible to sequence the entire genomes of multiple individuals from experimental populations. This allows the adaptation of a population at the genetic and phenotypic levels to be linked in ways that have simply not been possible in the past, offering unparalleled opportunities beyond simply describing the link between genotype and phenotype, and extending to understanding how this mapping could evolve (for example, Velicer et al., 2006). The use of genomics in experimental evolution may finally allow comparative and experimental literature on adaptation to be linked directly, expanding our ability to interpret the outcomes of particular experiments and to understand general patterns that emerge from population genetic type data. Experimental studies will be an essential component in providing a complete under- standing of both the importance that selection for evolvability has played in moulding the genomes of organisms in the past and the consequences of such characteristics for the future adaptive potential of species.

\section{Acknowledgements}

We are grateful to Alex Hall for comments on an earlier version of this manuscript and to Nick Barton and Sean Nee for discussion of the ideas. Two referees provided thoughtful criticism, which has improved the manuscript immeasurably. SC is supported by NERC.

\section{References}

Bell G (1982). The Masterpiece of Nature. Croom Hem: London and Canberra.

Bjedov I, Tenaillon O, Gerard B, Souza V, Denamur E, Radman $\mathrm{M}$ et al. (2003). Stress-induced mutagenesis in bacteria. Science 300: 1404-1409.

Chao L, Cox EC (1983). Competition between high and low mutating strains of Escherichia coli. Evolution 37: 125-134.

Charlesworth B, Barton NH (1996). Recombination load associated with selection for increased recombination. Genet Res 67: 27-41.

Colegrave N (2002). Sex releases the speed limit on evolution. Nature 420: 664-666.

Colegrave N, Buckling A (2005). Microbial experiments on adaptive landscapes. BioEssays 27: 1167-1173.

Colegrave N, Kaltz O, Bell G (2002). The ecology and genetics of fitness in Chlamydomonas. VIII. The dynamics of adaptation to novel environments after a single episode of sex. Evolution 56: $14-21$.

Collins S, de Meaux J, Acquisti C (2007). Adaptive walks towards a moving optimum. Genetics 176: 1089-1099.

Cox EC, Gibson TC (1974). Selection for high mutation rates in chemostats. Genetics 77: 169-184.

de Visser JAGM, Zeyl CW, Gerrish PJ, Blanchard JL, Lenski RE (1999). Diminishing returns from mutation supply rate in asexual populations. Science 283: 404-406.

Earl DJ, Deem MW (2004). Evolvability is a selectable trait. Proc Natl Acad Sci USA 101: 11531-11536.

Erill I, Campoy S, Mazon G, Barbe J (2006). Dispersal and regulation of an adaptive mutagenesis cassette in the bacteria domain. Nucleic Acids Res 34: 66-77.

Gerrish PJ, Lenski RE (1998). The fate of competing beneficial mutations in an asexual population. Genetica 103: 127-144.

Goddard MR, Godfray HC, Burt A (2005). Sex increases the efficacy of natural selection in experimental yeast populations. Nature 434: 636-640.

Grimberg B, Zeyl C (2005). The effects of sex and mutation rate on adaptation in test tubes and to mouse hosts by Saccharomyces cerevisiae. Evolution 59: 431-438.

Hansen TF (2006). The evolution of genetic architecture. Annu Rev Ecol Evol Syst 37: 123-157.

Jarosz DF, Beuning PJ, Cohen SE, Walker GC (2007). Y-family DNA polymerases in Escherichia coli. Trends Microbiol 15: 70-77.

Johnson T (1999a). The approach to mutation-selection balance in an infinite asexual population, and the evolution of mutation rates. Proc R Soc London B Biol Sci 266: 2389-2397.

Johnson T (1999b). Beneficial mutations, hitchhiking and the evolution of mutation rates in sexual populations. Genetics 151: 1621-1631.

Kaltz O, Bell G (2002). The ecology and genetics of fitness in Chlamydomonas. XII. Repeated sexual episodes increase rates of adaptation to novel environments. Evolution 56: 1743-1753.

Kirschner M, Gerhart J (1998). Evolvability. Proc Natl Acad Sci USA 95: 8420-8427. 
Kopp M, Hermisson J (2006). The evolution of genetic architecture under frequency-dependent disruptive selection. Evolution 60: 1537-1550.

LeClerc JE, Li BG, Payne WL, Cebula TA (1996). High mutation frequencies among Escherichia coli and Salmonella pathogens. Science 274: 1208-1211.

Martin G, Elena SF, Lenormand T (2007). Distributions of epistasis in microbes fit predictions from a fitness landscape model. Nature Genet 39: 555-560.

Maynard Smith J (1978). The Evolution of Sex. Cambridge University Press: Cambridge.

Metzgar D, Wills C (2000). Evidence for the adaptive evolution of mutation rates. Cell 101: 581-584.

Montville R, Froissart R, Remold SK, Tenaillon O, Turner PE (2005). Evolution of mutational robustness in an RNA virus. PloS Biol 3: 1939-1945.

Moore FB, Rozen DE, Lenski RE (2000). Pervasive compensatory adaptation in Escherichia coli. Proc $R$ Soc London B Biol Sci 267: 515-522.

Moxon ER, Rainey PB, Nowak MA, Lenski RE (1994). Adaptive evolution of highly mutable loci in pathogenic bacteria. Curr Biol 4: 24-33.

Narra HP, Ochman H (2006). Of what use is sex to bacteria? Curr Biol 16: R705-R710.

Nee S, Maynard Smith J (1990). The evolutionary biology of molecular parasites. Parasitology 100: S5-S18.

Oliver A, Canton R, Campo P, Baquero F, Blazquez J (2000). High frequency of hypermutable Pseudomonas aeruginosa in cystic fibrosis lung infection. Science 288: 1251-1253.

Poole AM, Phillips MJ, Penny D (2003). Prokaryote and eukaryote evolvability. Biosystems 69: 163-185.

Poon A, Chao L (2004). Drift increases the advantage of sex in RNA bacteriophage Phi 6. Genetics 166: 19-24.

Redfield RJ (2001). Do bacteria have sex? Nat Rev Genet 2: 634-639.

Renaut S, Replansky T, Heppleston A, Bell G (2006). The ecology and genetics of fitness in Chlamydomonas. XIII. Fitness of long-term sexual and asexual populations in benign environments. Evolution 60: 2272-2279.

Rosenberg SM, Thulin C, Harris RS (1998). Transient and heritable mutators in adaptive evolution in the lab and in nature. Genetics 148: 1559-1566.

Sanjuan R, Cuevas J, Furió V, Holmes E, Moya A (2007). Selection for robustness in mutagenized RNA viruses. PloS Genet 3: e93.
Sanjuan R, Cuevas JM, Moya A, Elena SF (2005). Epistasis and the adaptability of an RNA virus. Genetics 170: 1001-1008.

Sanjuan R, Elena SF (2006). Epistasis correlates to genomic complexity. Proc Natl Acad Sci USA 103: 14402-14405.

Shaver AC, Dombrowski PG, Sweeney JY, Treis T, Zappala RM, Sniegowski PD (2002). Fitness evolution and the rise of mutator alleles in experimental Escherichia coli populations. Genetics 162: 557-566.

Sniegowski PD, Gerrish PJ, Johnson T, Shaver A (2000). The evolution of mutation rates: separating causes from consequences. BioEssays 22: 1057-1066.

Sniegowski PD, Gerrish PJ, Lenski RE (1997). Evolution of high mutation rates in experimental populations of E. coli. Nature 387: 703-705.

Sniegowski PD, Murphy HA (2006). Evolvability. Curr Biol 16: R831-R834.

Tenaillon O, Le Nagard H, Godelle B, Taddei F (2000). Mutators and sex in bacteria: conflict between adaptive strategies. Proc Natl Acad Sci USA 97: 10465-10470.

Travisano M, Mongold JA, Bennett AF, Lenski RE (1995). Experimental tests of the roles of adaptation, chance, and history in evolution. Science 267: 87-90.

Velicer GJ, Raddatz G, Keller H, Deiss S, Lanz C, Dinkelacker I et al. (2006). Comprehensive mutation identification in an evolved bacterial cooperator and its cheating ancestor. Proc Natl Acad Sci USA 103: 8107-8112.

Wagner A (2005). Robustness, evolvability, and neutrality. FEBS Lett 579: 1772-1778.

Wagner GP, Altenberg L (1996). Perspective: complex adaptations and the evolution of evolvability. Evolution 50: 967-976.

Weinreich DM, Delaney NF, DePristo MA, Hartl DL (2006). Darwinian evolution can follow only very few mutational paths to fitter proteins. Science 312: 111-114.

Wilke CO, Adami C (2001). Interaction between directional epistasis and average mutational effects. Proc $R$ Soc London $B$ Biol Sci 268: 1469-1474.

Wilke CO, Wang JL, Ofria C, Lenski RE, Adami C (2001). Evolution of digital organisms at high mutation rates leads to survival of the flattest. Nature 412: 331-333.

Williams GC (1996). Adaptation and Natural Selection. Princeton University Press: Princeton.

Zeyl C, Bell G (1997). The advantage of sex in evolving yeast populations. Nature 388: 465-468. 